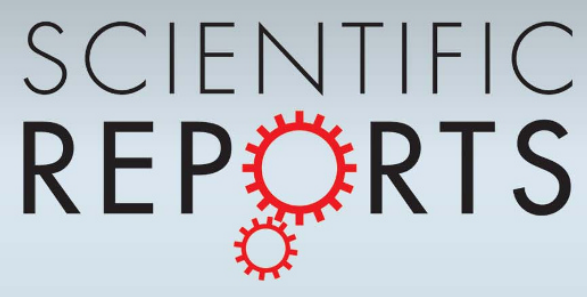

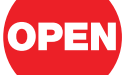

SUBJECT AREAS: GENERAL PHYSICS

FLUIDS

MODELLING AND THEORY

PHYSICS

Received

13 September 2011

Accepted

13 December 2011

Published

9 January 2012

Correspondence and requests for materials should be addressed to

H.S. (hsoumerai@ gmail.com)

\section{Analytical method of predicting turbulence transition in pipe flow}

\author{
Henri Soumerai \& Brigid E. Soumerai-Bourke
}

H.E.D.Soumerai \& Associates.

The present analysis is based on the complete first law flow equation i.e. it includes the kinetic energy term that was neglected in earlier papers. It is shown that the results obtained with the proposed equations, derived from an Entropy Maximizing Principle, agree quite well with reliable data published in the scientific/technical literature.

he validity of the "theorem" of minimum entropy production (per unit time) was assessed in Refs 1 and 2 for the well documented case of single - phase fluid tube flow bifurcation from laminar to turbulent regimes. The analysis led to the following two main conclusions: 1) This theorem is not generally valid. 2) The stable type of flow regime is associated with a maximum specific (per unit mass) entropy change. More general broader statements of optimality in thermodynamics are now available in ${ }^{3}$.

In order to avoid the range of $R e$ in the so called "transition region" the analysis in ${ }^{1,2}$ was limited to Reynolds numbers one order of magnitude above the estimated critical Reynolds number $R_{c r}$ for the transition to turbulent flow and the kinetic energy term of the first law flow equation was neglected in line with normal engineering practice.

In the present report the analysis is carried out down to $R e_{\infty}<R e_{c r}$ and based on the complete flow equation i.e. it includes the kinetic term. As a result a new independent variable: the relative tube length or its inverse $L / D$ or $D / L$ as well as two parameters: the laminar and turbulent kinetic energy factors $M_{l}$ and $M_{t}$ respectively, appear in the analysis and its solution. As the kinetic energy factors, or multipliers $M$ play a key role in our analysis, a few words may be useful to clarify the definition of these parameters and provide a brief explanation on the methods adopted to assess their values under fully developed flow conditions for Newtonian fluids flowing in straight horizontal circular pipes.

The kinetic energy term $V^{2} / 2 g$ is only valid in the idealized world of inviscid fluid mechanics; due to the absence of friction the average velocity $V$ also represents the local velocity that remains constant within the whole tube flow area. With real Newtonian fluids the local velocity is no longer constant and diminishes to zero at the pipe innerwall for both flow regimes. In the specific case of laminar regimes the actual local velocities could be determined accurately on the basis of Newton's laws of mechanics and from this a constant factor $M_{l}=2$ determined to compute the correct kinetic energy term according to $2\left(V^{2} / 2 g\right)$. For turbulent regimes no exact theories are available to determine $M_{t}$ (as well $f_{t}$ ) and we depend entirely on experiments. Fortunately $M_{t} \approx 1$ and its impact on the prediction of $R e_{c r}$ is negligible compared to the laminar kinetic energy factor $M_{l}$.

The same end results are obtained $\mathrm{in}^{4}$ and the present report; the basic difference between these two communications lies in the method adopted to predict the critical Reynolds number $R e_{c r}$. $\operatorname{In}^{4}$ a quasi "empirical" approach was selected based on numerous plots produced with EXCEL. In the present report a purely analytical method was adopted based on a single premise: the extension of the validity of the Entropy Maximizing Principle proposed in ${ }^{1,2}$ to any Reynolds number, including the singularity at $R e_{\infty}$ where $D / L=\mathrm{O}$ and $L / D \rightarrow \infty$.

Although this topic may be of interest to some physicists, the theoretical prediction of the critical Reynolds number should prove particularly useful to scientists and engineers engaged in fluid mechanics and heat transfer mainly for several reasons that can be highlighted as follows. Soon after he demonstrated the existence of a "sinuous flow" regime and proposed an excellent estimate of $R e_{c r}=2200$ for smooth circular tubes, Reynolds derived what became known as the"Reynolds analogy", This shows that the internal hear transfer coefficient $h$ for a circular pipe is directly proportional to the friction factor $f_{t}$. Since the $h_{t}$ is usually much larger than $h_{l}$ it is advantageous to operate in turbulent regimes. Therefore an advanced knowledge of $R e_{c r}$ for instance in such radically new applications as micro-channel heat exchangers is quite important ${ }^{4}$. 


\section{Results}

Analysis. The well known ${ }^{5}$ first law flow equation can be expressed as follows

$$
H=(L / D)\left(V^{2} / 2 g\right)[f+M(D / L)]
$$

where $H$ is the total head, $V$ the average velocity and $f$ the DarcyWeissbach friction factor. Equation (1) is valid for laminar with $f_{l}$, $M_{l}$ and $f_{t}, M_{t}$ for turbulent flow. It is convenient to define a dimensionless relative head

$$
H_{\text {rel }}=H / H_{\text {frict }, \infty}
$$

where $H_{\text {frict } \infty}$ is the head loss due only to friction at $R e_{\infty}$ where the laminar and turbulent friction factor curves intersect or

$$
H_{\text {frict }, \infty}=(L / D)\left(V^{2} / 2 g\right)\left[f_{\infty}\right]
$$

with

$$
f_{\infty}=f_{t}=f_{l} \text { at } R e=R e_{\infty}
$$

Since according to ${ }^{1,2,6 a}, H=T \Delta s$ and $H_{\text {frict } \infty}=T \Delta s_{\text {frict }, \infty}$ with $T$ and $\Delta s$ the absolute temperature and specific entropy change, it is clear that under constant temperature conditions

$$
H_{r e l}=\Delta S_{r e l}
$$

and in view of (1) to (5)

$$
H_{\text {rel }}=\Delta S_{\text {rel }}=[f+M(D / L)] / f_{\infty}
$$

The relative dimensionless entropy change $\Delta s_{r e l}$ is used from here on instead of $H_{\text {rel }}$. and it follows from (6)

$$
\begin{aligned}
& \Delta S_{r e l, l}=\left[f_{l}+M_{l}(D / L)\right] / f_{\infty} \\
& \Delta S_{r e l, t}=\left[f_{t}+M_{t}(D / L)\right] / f_{\infty}
\end{aligned}
$$

By treating both $\Delta s_{r e l, t}$ and $\Delta s_{r e l, l}$ as independent mathematical functions, it is permissible to set

$$
\Delta S_{r e l, l}=\Delta S_{r e l, t}
$$

yielding with (7) and (8)

$$
f_{t}+M_{t}(D / L)=f_{l}+M_{l}(D / L)
$$

noting that $(10)$ is now independent of $f_{\infty}$; and can be reduced to

$$
D / L=\left(f_{t}-f_{l}\right) /\left(M_{l}-M_{t}\right)
$$

It is interesting to note that as long as $M_{l}>M_{t}$ and $\left(M_{l}-M_{t}\right)$ is a finite number, then (11) yields $D / L=0$, therefore $L / D \rightarrow \infty$ at $R e_{\infty}$, as it should.

If $M_{l}$ and $M_{t}$ were exactly equal (11) would yield $D / L=0 / 0$; however the two kinetic terms in (10) would cancel each other and the result would be: $f_{l}=f_{t}=f_{\infty}=f_{c r}$ and $R e_{\infty}=R e_{c r}$. It is due to the fact that $M_{l} / M_{t}=2>1$ in the case of smooth circular tubes with fully developed flow at inlet that $R e_{c r} / R e_{\infty}=2.55>1$ as shown in $^{4}$.

Minimum $R e_{c r,} f d$ with fully developed flow at inlet of circular pipes. According to $M_{l}=2$ and $M_{t} \approx 1$; introducing these values in (11) then the expression of $D / L$ thus obtained in both sides of (10) yields the same equation for $\Delta s_{r e l, l}$ and $\Delta s_{r e l}, t$

$$
\Delta S_{r e l}=\left[2 f_{t}-f_{l}\right]
$$

As this mathematical function of $R e$ has a maximum, we postulate that $R e_{\infty}$ occurs there in order to maximize the entropy change according to ${ }^{1,2}$. Therefore $R e_{c r}$ can be determined from

$$
\mathrm{d} \Delta S_{r e l} / \mathrm{d} R e=0
$$

or, with (12)

$$
\mathrm{d} f_{t} / \mathrm{d} R e=(1 / 2) \mathrm{d} f_{l} / \mathrm{d} R e=\left(M_{t} / M_{l}\right) \mathrm{d} f_{l} / \mathrm{d} R e
$$

which means that the bifurcation to turbulent flow occurs at $R e=R e_{c r}$ where, in a linear coordinate system, the slope of $f_{t}$ is one half that of $f_{l}$.

Maximum $R e_{c r}$, fud with fully undeveloped flow at inlet of circular pipes. This occurs when $M_{l} \rightarrow 1$, for instance 1.01 or $1.001 \ldots$ and $M_{t}=1$. Following the same procedure as in the previous case yields as a limit

$$
\mathrm{d} f_{t} / \mathrm{d} R e=(1) \mathrm{d} f_{l} / \mathrm{d} R e=\left(M_{t} / M_{l}\right) \mathrm{d} f_{l} / \mathrm{d} R e
$$

The slope of $f_{t}$ is identical to that of $f_{l}$ in this limiting case, meaning that $R e_{c r, f u d}$ must be significantly larger than $R e_{c r} f d$. This is corroborated to some extent by the following excerpts from the abstract of presented at the 2007 Annual Review of Fluid Mechanics: "Experiments on pipe flow,.... show that triggering turbulence depends sensitively on initial conditions."

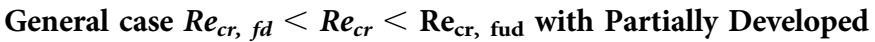
Flow at pipe Inlet. It is easy to show that (14) and (15) are individual cases of the following more general equations

$$
\begin{gathered}
\Delta S_{r e l}=\left(M_{l} f_{t}-M_{t} f_{l}\right) /\left(M_{l}-M_{t}\right) \\
\mathrm{d} f_{t} / \mathrm{d} R e=\left(M_{t} / M_{l}\right) \mathrm{d} f_{l} / \mathrm{d} R e
\end{gathered}
$$

for circular pipes:

$$
M_{l}=2 \rightarrow \approx 1, M_{t} \approx 1 \text { and } M_{t} / M_{l}=1 / 2 \rightarrow \approx 1
$$

It is noteworthy that the above equations were derived without using any explicit equations or correlations for $f_{l}$ or $f_{t}$, on the basis of an "Entropy Maximizing Principle", equation (13), and should therefore be applicable with any reliable empirical correlation of $f_{t}$ and valid for any pipe size, including micro tubes and channels.

Prediction of $\boldsymbol{R e}_{c r}$, $f$ with fully developed flow at inlet of smooth circular pipes. To validate the proposed theoretical equations it is necessary to compute $R e_{c r}$ with equation (14) and demonstrate that the critical Reynolds numbers obtained in this manner substantially agree with the most reliable data published in the scientific/technical literature. As the exact equation of $f_{l}$ in terms of $R e$ is well known the right hand side of (14) presents no problem. For the left hand side we are faced with the following challenge: which one among the many empirical correlations proposed for the turbulent friction factor is best suited to our present purpose? Koo's excellent empirical correlation $^{7}$ based on his 1932 thesis at MIT is selected here for the following reasons.

The values of $f_{t}$ obtained with four different correlations are shown in [5b]. Two of them, are clearly more accurate than the others, namely Petukhov's ${ }^{9}$ and Koo' ${ }^{7}$ correlations with the following Re validity limits 10,000 to $3,000,000$ and 3,000 to 300,000 respectively. Since we focus in this study on Re below 10,000, Koo's correlation is the better choice; furthermore the maximum deviation between these two correlations being less than 1.5\%, Petukhov's correlation is contained within the $\pm 5 \%$ accuracy band of Koo's correlation based on 1380 most reliable test resuls.

By introducing in (14) Koo's correlation for $f_{t}$ and the exact equation of $f_{l}$ for the laminar regime, we obtain $\mathrm{d}(0.0056+0.5$ $\left.R e^{-0,32}\right) / \mathrm{d} R e=0.5 \mathrm{~d}\left(64 R e^{-1}\right) / \mathrm{d} R e$, yielding $R e^{0.68}=200$ resulting in $R e_{c r}=2400$. In order to make allowance for its $\pm 5 \%$ accuracy, it is only necessary to multiply Koo's correlation by 0.95 and 1.05 , resulting in a possible range of $R e_{c r}=2160$ to 2610 .

The uppermost value $R e_{c r}=2610<3000$, as it should, since this is the lower validity limit of Koo's correlation for turbulent flow based on tests with fully developed flow at tube inlet extending down to $R e=3000$.

The lowest value $R e_{c r}=2160 \approx 2200$, below which the flow remains laminar regardless of inlet conditions according to ${ }^{5,6 b}$. It is noteworthy that Osborne Reynolds, following his discovery of 


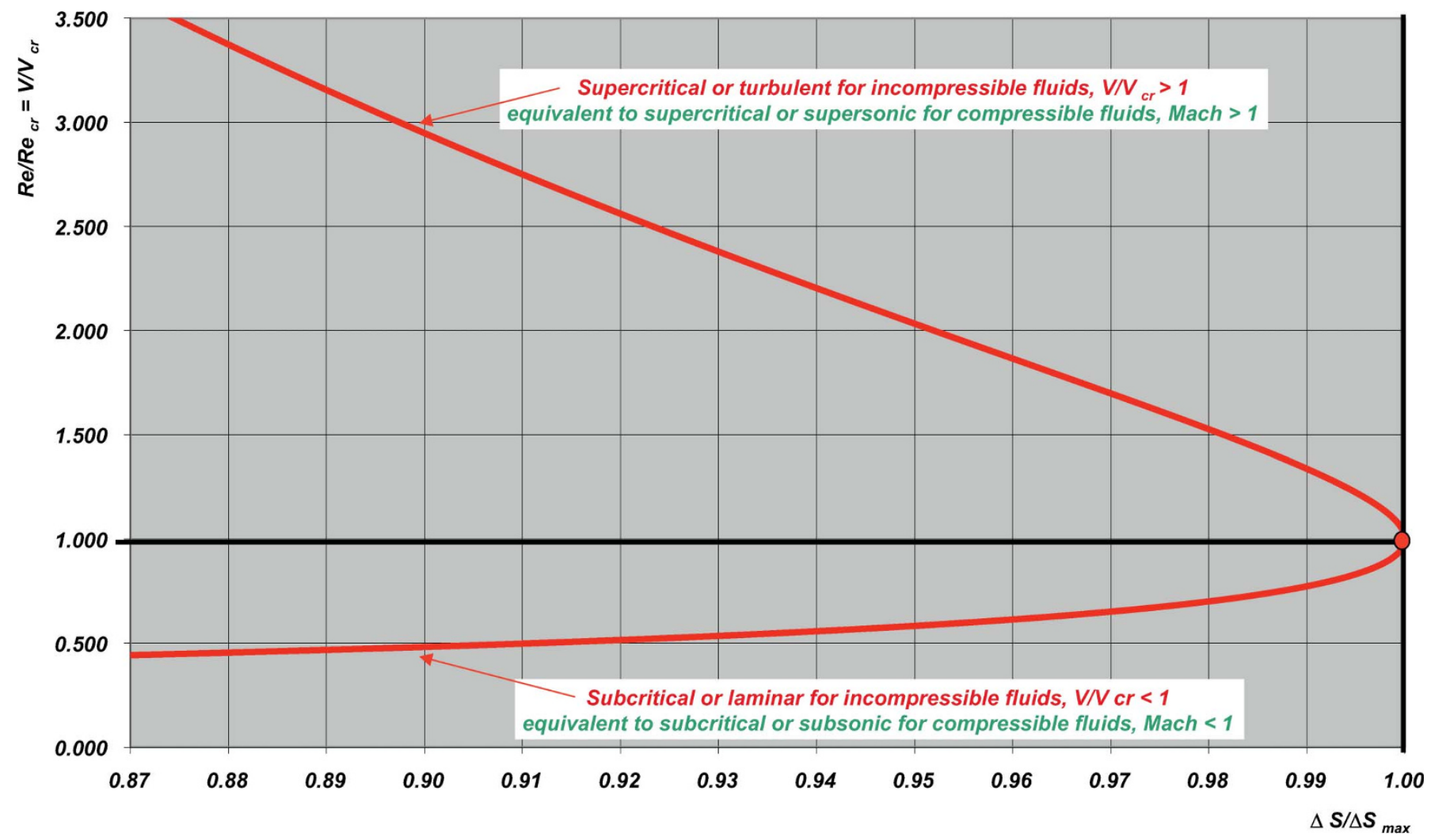

Figure $1 \mid$ FANNO Flow Curve for Incompressible Fluids.

Sinuous Flows ${ }^{9}$ in 1884, proposed a value of 2200 for what was later called the Reynolds Number!

These positive results validate the proposed basic equation (17) with which they were obtained.

Conversely, if one accepts this conclusion and remembers that (17) was derived from the application of the Principle of Entropy Maximization (13), then the principle itself has been validated.

Estimate of $\boldsymbol{R} \boldsymbol{e}_{c r}$, fud with fully undeveloped flow at inlet of smooth circular pipe. The impact of the laminar factor $M_{l}$ on $R e_{c r}$ can be roughly estimated (as Koo's correlation based on tests with fully developed inlet flow is no longer as accurate) with (17) and 0.95. $f_{t}$ thus obtaining $R e^{0.68} \approx 420$ and $R e_{c r} \approx 7300$.

This estimate is sufficiently accurate to demonstrate the significant impact of $M_{l}$ on $R e_{c r}$ and to explain why $R e_{c r}>3000$ have been reported with smooth tubes as indicated in the 2009 Moody Chart shown in ${ }^{5}$.

\section{Discussion}

A most succinct definition ${ }^{11}$ of engineering thermodynamics is: The Science of Energy and Entropy. Accordingly our analysis moved from conventional Fluid Mechanics to Classical Thermodynamics from equation (7) onwards and led, based on an entropy maximization principle, to the main equations (9), (11), (12), (13), (16) and (17).

At some point it became clear to the authors that equation (16) would lead to an incompressible fluid Fanno flow curve. Indeed all individual curves of $\Delta s_{r e l}$ in terms of $R e$ produced with (16) can be reduced to a single common curve by changing the coordinates as follows: Replace $\Delta s_{r e l}$ by $\Delta s_{r e l} /\left(\Delta s_{r e l}\right)_{\text {max }}$ with $\left(\Delta s_{r e l}\right)_{\text {max }}$ obtained with (16) at $R e_{c r}$ and substitute $V / V_{c r}=R e / R e_{c r}$ for $R e$. The curve obtained in this manner shown in Fig. 1 (reproduced from ${ }^{4}$ ) is a FANNO FLOW curve according to the Extended Definition of Fanno Flow $\mathrm{in}^{12}$. The notes in green in figure 1 show the similarity with the more complex transition from sub-sonic to super-sonic flow regimes.
One of the important features of classical thermodynamics is that its laws are independent of the size and geometry of the systems under consideration. This fact permits extending the application of (17) beyond smooth tubes to include non-smooth pipes and constant cross section channels of any shape and size i. e. including micro tubes and channels used in micro heat exchangers ${ }^{3}$, provided suitably accurate $f_{t}$ and $f_{l}$ correlations as well as inlet parameters $M_{l}$ and $M_{t}$ are available with fully developed flow at pipe inlet.

The need and means of suppressing the laminar flow regimes in order to reduce the size of compact tubular heat exchangers is illustrated with the help of an internally finned tube design (INF) of rather complex geometry compared to standard circular pipes $i^{4}$. This INF design patented in the 1940's was basically a forerunner of modern micro-channels heat exchangers.

For scientists/engineers active in thermal sciences the following list of key words may be helpful to identify the novel or little known features included in this short report: Fluid flow thermodynamics; Maximizing entropy; Incompressible FANNO flow; laminar kinetic energy factor and; Impact of relative tube length.

1. Soumerai, H. On the Application of an "Entropy Maximizing Principle" in Flow Regime Prediction, International Communications Heat and Mass Transfer, 14, 303-312 (1987).

2. Soumerai, H. Thermodynamic aspects of Adiabatic and Diabatic Tube Flow Regime Transition - Single Phase Fluids, as paper number 1384 published in AIAA/ASME $4^{\text {th }}$ Joint Thermophysics and Heat Transfer Conference June 2 - 4, 1986/ Boston Mass.

3. Bejan, A. \& Lorente, S. The constructal law of design and evolution in nature, Philosophical Transactions of the Royal Society B, 365, 1335-47 (2010).

4. Soumerai, H. Application of a Novel Fanno Flow Equation, International Institute of Refrigeration's Web site, scheduled for publication in 2011.

5. 2009 ASHRAE Handbook - Fundamentals (I-P Edition). American Society of Heating, Refrigerating and Air-Conditioning Engineers, Inc. Online version available at: http://www.knovel.com/web/portal/browse/display?_EXT_ KNOVEL_DISPLAY_bookid $=2554 \&$ VerticalID $=0$, Chapter 3 Fluid Flow.

6. Soumerai, H. Practical Thermodynamic Tools for Heat Exchanger Design Engineers, a) Chapt. 2, Sect. 2.2 From Reversible to Irreversible Fluid Flow p.20, 
b) Chapt 3, Sect. 3.2 Laminar and Turbulent Flow Regimes p. 26, 1987, Wiley Interscience Publications, John Wiley \& Sons.

7. Koo, E. Mechanisms of isothermal and non-isothermal flow of fluids in pipes, Sc D Thesis, Mass. Inst. of Technology, Dept. of Chemical Engineering, (1932).

8. Eckhardt, B., Schneider, T., Hof, B. \& Westerweel, J. Turbulence Transition in Pipe Flow. Annual Review of Fluid Mechanics 39; 447-468 (2007).

9. Petukhov, B. S. Heat Transfer and Friction in Turbulent Pipe Flow with Variable Physical Properties in Advances in Heat Transfer, J. P. Hartnett and T. F. Irvine, J,R.(EDS.), 6, 503-564, Academic Press, New York, 1970.

10. Reynolds, O. On the Two Motions of Water, Proc R. Inst Great Britain, 1884.

11. Sonntag, R., Gordon, J. \& Van Wylen. Introduction to Thermodynamics, Classical and Statistical, $2^{\text {nd }}$ ed., Chap.2, p. 16, Wiley, New York, 1982.

12. Webster's Extended Definition of Fanno Flow, May 2011, www.webster-onlinedictionary.org.

\section{Author contributions}

Both authors contributed equally to the writing and both reviewed the manuscript.

\section{Additional information}

Competing financial interests: The authors declare no competing financial interests.

License: This work is licensed under a Creative Commons

Attribution-NonCommercial-ShareAlike 3.0 Unported License. To view a copy of this license, visit http://creativecommons.org/licenses/by-nc-sa/3.0/

How to cite this article: Soumerai, H. \& Soumerai-Bourke, B.E. Analytical method of predicting turbulence transition in pipe flow. Sci. Rep. 2, 214; DOI:10.1038/srep00214 (2012). 